\title{
Performance evaluation of optical platelet counting of BC-6000Plus automated hematology analyzer
}

\author{
Xuehua Hü, Yuqun Tang", Mingyi Li", Chunyun Fu, Zuoxin Deng, Wenting Tang, Huideng Zhou, \\ Yan Chen, Yingfeng Li
}

Medical Science Laboratory, The Maternal and Child Health Hospital of Guangxi Zhuang Autonomous Region, Nanning, China

Contributions: (I) Conception and design: Y Li; (II) Administrative support: H Zhou, Y Chen; (III) Provision of study materials or patients: C Fu, W Tang, Z Deng; (IV) Collection and assembly of data: X Hu; (V) Data analysis and interpretation: Y Tang, M Li; (VI) Manuscript writing: All authors; (VII) Final approval of manuscript: All authors.

"These authors contributed equally to this work.

Correspondence to: Yingfeng Li. Medical Science Laboratory, The Maternal and Child Health Hospital of Guangxi Zhuang Autonomous Region, no.59 Xiangzhu Road, Nanning 530003, China. Email: lyf1517@126.com.

Background: The BC-6000Plus (Mindray, Shenzhen, China) is a recently developed hematology analyzer that utilizes fluorescent technology. Based on fluorescent nucleic acid stain and optical detection, the optical platelet counting (PLT-O) on the BC-6000Plus has strong anti-interference potential in platelet (PLT) detection. Its Auto $8 \times$ PLT-O Counting Tech can be automatically triggered in low-PLT samples, which enables the PLT-O on the BC-6000Plus to count low PLT more efficiently. Here, we evaluated the performance of the BC-6000Plus automated hematology analyzer in optical PLT counting.

Methods: The basic features (including blank counting, carryover, trueness, and accuracy) of the BC6000Plus for PLT counting were evaluated according to the Analytical Quality Specifications for Routine Tests in Clinical Hematology (WST 406-2012). Low-PLT samples with a PLT count of below $100 \times 10^{9} / \mathrm{L}$ were selected for repeatability tests. Meanwhile, the potential correlations of BC-6000Plus with the XN-L 350 and manual microscopy within different PLT ranges or under interferences of small red blood cells (RBCs) or PLT aggregation were analyzed.

Results: The PLT-O on BC-6000Plus met the technical requirements of PLT counting in terms of blank count, carryover, trueness, and accuracy. The repeatability of the enhanced mode (PLT-O $8 \times$ ) on the BC6000Plus was better than that of the XN-L 350 in three low PLT count ranges, including 10-20, 20-60, and 60-100 $\left(\times 10^{9} / \mathrm{L}\right)$. Under the interference-free conditions, the BC-6000Plus correlated well with the XN-L 350 in different PLT counting ranges. Under the interferences of small RBCs and PLT aggregation, the PLT-O on BC-6000Plus correlated better with microscopy than with the platelet impedance count (PLT-I).

Conclusions: The PLT-O on BC-6000Plus can meet the technical requirements of PLT counting in terms of blank counting, carryover rate, trueness, and accuracy. The PLT-O $8 \times$ has good repeatability, correlates well with the XN-L 350, and demonstrates good anti-interference ability. It can thus meet the needs of blood cell analysis in clinical settings.

Keywords: Optical platelet counting (PLT-O); enhanced optical platelet counting (enhanced PLT-O); low platelet count (low PLT count); platelet aggregation (PLT aggregation)

Submitted Dec 21, 2020. Accepted for publication Mar 22, 2021.

doi: 10.21037/atm-21-691

View this article at: http://dx.doi.org/10.21037/atm-21-691 


\section{Introduction}

Numerous advancements in blood cell analysis, including platelet (PLT) detection, have been made in recent years. The main procedures for PLT counting include microscopy, fully automated hematology analyzer, and flow cytometry-based methods recommended by the International Council for Standardization in Hematology (ICSH) $(1,2)$. Immunofluorescence detection by flow cytometry is the international reference method recommended by the ICSH; however, it requires highly skillful and competent personnel and is quite expensive, which limits its application in clinical laboratories (3). Microscopy is often used for rechecking PLT counts, especially when interfering factors such as small red blood cells (RBCs) and PLT aggregation are present, for which microscopy offers a more visible and specific option. However, this method also has many limitations: (I) the tester must have adequate knowledge of cellular morphology; (II) the test result is highly subjective; (III) the intertester difference is larger; (IV) the test is difficult, time-consuming, and has poor reproductivity (4). Fully automated hematology analyzers for PLT detection have been widely used because they are fast, easy to use, and inexpensive. PLT counting on hematology analyzers can be performed using different methods (5): impedance (PLT-I), optical (PLT-O), and fluorescent nucleic acid dye (PLT-F). The BC-6000Plus fully automated hematology analyzer (Mindray, Shenzhen, China) uses fluorescent nucleic acid dye to stain PLTs to obtain optical PLTs (i.e., PLT-O); in addition, to overcome the poor coefficient of variation of PLT-I in low platelet samples, the analyzer can automatically prolong counting time up to 8 -fold (PLT-O $8 \times$ ) to collect more PLT particles if the PLT count is below the cutoff value (6). Previous study (5) has demonstrated that BC-6000Plus has superior association and consistency for normal platelet counts, but it didn't show the performance of BC-6000Plus PLT-O under the interference of small RBCs or PLT aggregation. In our current study, we evaluated the technical performance of BC-6000Plus in terms of blank count, carryover, trueness, and accuracy. Meanwhile, we verified its repeatability in determining low PLTs. Finally, we compared its performance with that of conventional tests in PLT determination in routine blood samples and in blood samples with interference. We present the following article in accordance with the MDAR checklist (available at http://dx.doi.org/10.21037/atm-21-691).

\section{Methods}

\section{Equipment and reagents}

The BC-6000Plus fully automated hematology analyzer and its accompanying reagents, quality control products, and calibrators were manufactured by Mindray Bio-Medical Electronics (Shenzhen, China). The XN-L 350 hematology analyzer and its supporting reagents, quality control products, and calibrators were manufactured by Sysmex Medical Electronics Co. (Kobe, Japan).

\section{Sources of EDTA-K $\mathrm{K}_{2}$-anticoagulated fresh whole-blood samples}

A total of 122 routine blood samples were collected in the Clinical Laboratory of the Maternal and Child Health Hospital of Guangxi Zhuang Autonomous Region from March 2019 to November 2019. The distribution of PLT counts in these samples was as follows: $>500 \times 10^{9} / \mathrm{L}$, $\mathrm{n}=10 ;(200-500) \times 10^{9} / \mathrm{L}, \mathrm{n}=20 ;(100-200) \times 10^{9} / \mathrm{L}, \mathrm{n}=20$; $(60-100) \times 10^{9} / \mathrm{L}, \mathrm{n}=10$; and $<60 \times 10^{9} / \mathrm{L}, \mathrm{n}=10$; in addition, there were 20 samples with small RBC fragments and 10 samples with EDTA-dependent PLT aggregation.

This study was approved by the ethics committee of The Maternal and Child Health Hospital of Guangxi Zhuang Autonomous Region. (No. [2021]2-1). All procedures performed in this study involving human participants were in accordance with the Declaration of Helsinki (as revised in 2013). Informed consent was obtained from the patients.

\section{Study methods}

\section{Calibration and quality control of the hematology analyzers}

The BC-6000Plus and XN-L350 hematology analyzers were calibrated and verified using the supporting calibrators. The background and quality control tests were performed before the daily testing. The samples were tested before the background met the requirements and the quality control results were within the normal range.

\section{Background counting}

The diluent was used as the sample and tested on the analyzers three times, and the maximum of the three measurement values was used as the background count.

\section{Carryover}

A high-concentration EDTA- $\mathrm{K}_{2}$-anticoagulated whole- 
blood sample [white blood cell $(\mathrm{WBC})>90.0 \times 10^{9} / \mathrm{L}$, $\mathrm{RBC}>6.20 \times 10^{12} / \mathrm{L}$, hemoglobin $(\mathrm{HGB})>220 \mathrm{~g} / \mathrm{L}$, and PLT $\left.>900 \times 10^{9} / \mathrm{L}\right)$ was obtained. After the concentration was mixed well, three measurements were performed consecutively, and the measured values were set as H1, H2, and $\mathrm{H} 3$, respectively. Then, a low-concentration of EDTA$\mathrm{K}_{2}$-anticoagulated whole-blood sample $\left(\mathrm{WBC}<3.0 \times 10^{9} / \mathrm{L}\right.$, $\mathrm{RBC}<1.50 \times 10^{12} / \mathrm{L}, \mathrm{HGB}<50 \mathrm{~g} / \mathrm{L}$, and PLT $>30 \times 10^{9} / \mathrm{L}$ ) was obtained and consecutively measured three times, and the measured values were set as L1, L2, and L3, respectively. The carryover rate was calculated using the formula: carryover rate $=[(\mathrm{L} 1-\mathrm{L} 3) /(\mathrm{H} 3-\mathrm{L} 3)] \times 100 \%$.

\section{Verification of trueness}

The verification of trueness was based on item 5.6.2 of Analytical Quality Specifications for Routine Tests in Clinical Hematology (WST 406-2012). Ten fresh blood samples were used, and each sample was tested twice to calculate the mean value of the test results. The bias was calculated with the calibrated value as the standard.

\section{Verification of accuracy}

The verification of accuracy was based on item 5.9.2 of Analytical Quality Specifications for Routine Tests in Clinical Hematology (WST 406-2012). Five clinical samples with laboratory-calibrated values were obtained and tested once. The relative deviation of the test result from the calibration value was calculated for each sample.

\section{Verification of the repeatability for low PLT counting}

The repeatability for low PLT counting was verified based on item 6.5 of Hematology Analyzer (YY/T 0653-2017). For samples with low-PLT values, five samples were selected from each of the three ranges, including $10-\leq 20,>20-\leq 60$, and $>60-\leq 100 \times 10^{9} / \mathrm{L}$, and the repeatability was analyzed by using the BC-6000Plus and XN-L 350, respectively. Ten measurements were performed on each instrument with conventional method (enhanced PLT-O for BC6000Plus and PLT-O for XN-L 350), and the medians (M), interquartile ranges $(\mathrm{Q})$, ranges $(\mathrm{R})$, and robust coefficient of variation $[\mathrm{CV}(\%)][$ robust $\mathrm{CV}(\%)=$ ] were calculated for the ten replicate determinations of each sample. The 15 pairs of robust $\mathrm{CV}$ (\%) values corresponding to $\mathrm{BC}$ 6000 Plus and XN-L 350 were subjected to a paired $t$ test.

\section{Correlations among PLT measurements}

The potential correlations of BC-6000Plus test results with $\mathrm{XN}-\mathrm{L} 350$ and manual count results under different PLT ranges and different interference were compared.

\section{Statistical analysis}

Data analysis was achieved by using SPSS version 17.0 (SPSS Inc., Chicago, IL, USA). The Spearman correlation coefficient was used to assess the correlation between different detection methods.

\section{Results}

\section{Background counts}

The background counting was performed on a daily basis after power-on according to the requirements of WST 4062012. During the verification tests, the background PLT count was 0 in all samples, which met the requirement (PLT $\leq 10 \times 10^{9} / \mathrm{L}$ ) of item 5.1.1 of Quality Requirements for the Analytical Quality Specifications for Routine Tests in Clinical Hematology (WST 406-2012).

\section{Results of carryover verification}

A high-concentration clinical sample (EDTA- $\mathrm{K}_{2}-$ anticoagulated venous blood) was mixed well and measured three times. Then, a low-concentration clinical sample (EDTA- $\mathrm{K}_{2}$-anticoagulated venous blood) was mixed well and measured three times. The carryover rate was calculated using the following formula: carryover rate $=[(\mathrm{L} 1-\mathrm{L} 3) /$ $(\mathrm{H} 3-\mathrm{L} 3)] \times 100 \%$. It was found the carryover rates were below $0.05 \%$ in all samples, which met the requirement (CR $\leq 4.0 \%$ ) of item 5.2.1 of Quality Requirements for the Analytical Quality Specifications for Routine Tests in Clinical Hematology (WST 406-2012).

\section{Results of trueness verification}

The bias of PLT measurements was $0.74 \%$ among 10 fresh blood samples, which met the requirement (PLT measurement bias $\leq 6.0 \%$ ) of item 5.6.1 of Quality Requirements for the Analytical Quality Specifications for Routine Tests in Clinical Hematology (WST 406-2012).

\section{Results of accuracy verification}

The relative discrepancies of five clinical samples calibrated by the calibration laboratory were $3.54 \%, 1.52 \%, 3.55 \%$, $1.22 \%$, and $1.18 \%$, respectively, all of which were $\leq 20.0 \%$ 
Table 1 Results of PLT counting by two different instruments in fifteen samples with low PLT values

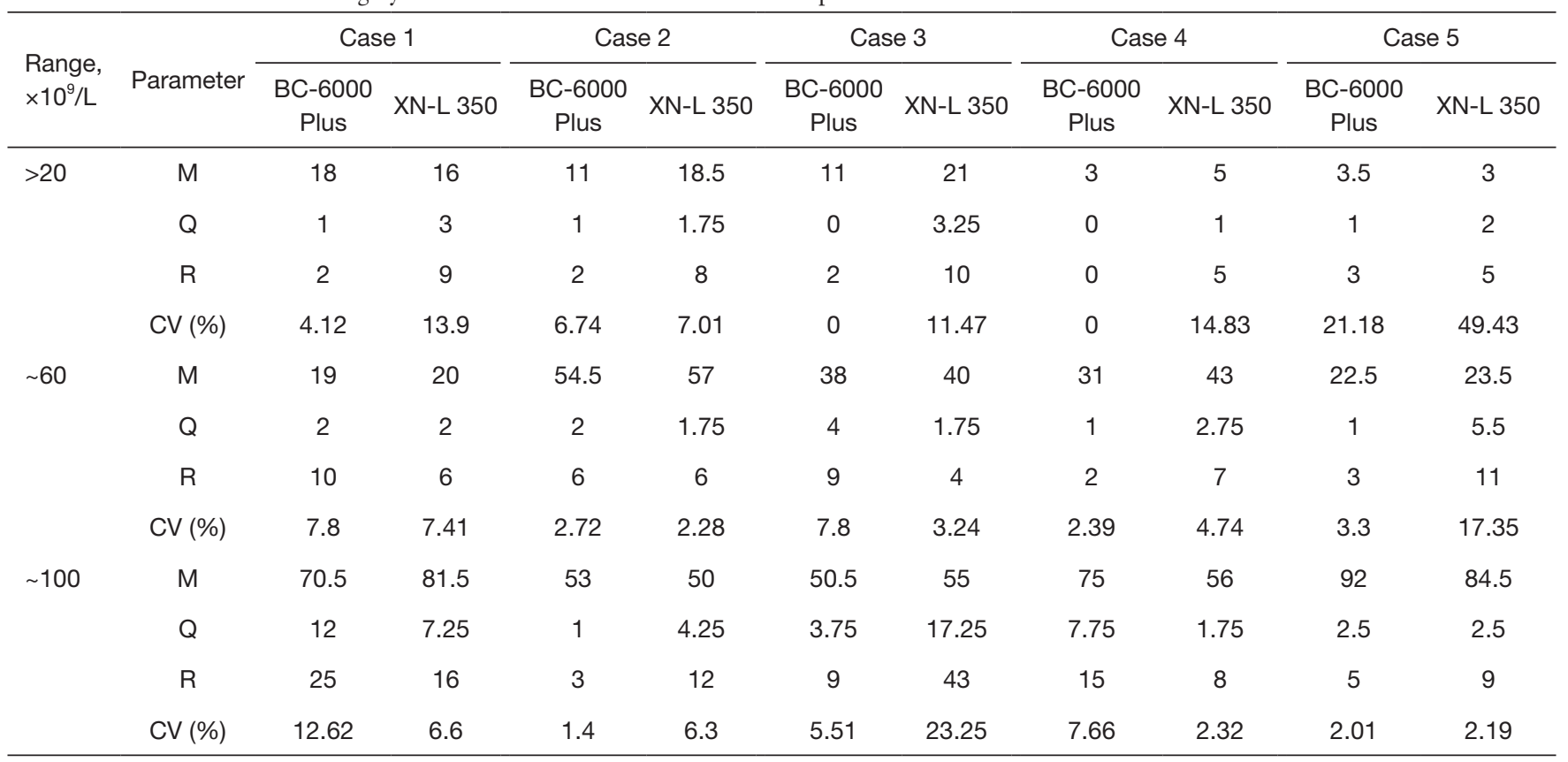

The paired $t$ test showed that the difference in CV (\%) between BC-6000Plus and XN-L 350 for low-PLT samples was statistically significant ( $t=2.275, P=0.039)$. $P L T$, platelet; $M$, medians; $Q$, interquartile ranges; $R$, ranges; $C V$, coefficient of variation.

and met the requirement (the accuracy shall be $\geq 80 \%$ ) of item 5.9.2 of Quality Requirements for the Analytical Quality Specifications for Routine Tests in Clinical Hematology (WST 406-2012).

\section{Repeatability for low PLT counting}

Five samples were selected from each of the three ranges, including $10-\leq 20,>20-\leq 60,>60-\leq 100$, and enhanced PLT-O was performed on BC-6000Plus. Indicators of repeatability included the medians $(M)$, interquartile ranges $(\mathrm{Q})$, ranges $(\mathrm{R})$, and robust $\mathrm{CV}(\%)$ (Table 1). Compared with the PLT-O on XN-L 350, the enhanced PLT-O on BC-6000Plus had better repeatability in detecting low-PLT samples, with a significantly smaller robust $\mathrm{CV}$ (\%).

\section{Correlations among PLT measurements}

Blood samples within four different PLT ranges [ $(0-$ $100)] \times 10^{9} / \mathrm{L},(100-200) \times 10^{9} / \mathrm{L},(200-500) \times 10^{9} / \mathrm{L}$, and $>500 \times 10^{9} / \mathrm{L}$ were selected for PLT-I and PLT-O assays by the BC-6000Plus, PLT-I and PLT-O assays by the XN-L 350 , and manual microscopy. The correlations of BC6000 plus with manual microscopy and the XN-L 350 are shown in Table 2, which shows that the BC-6000plus correlated better with the XN-L 350.

Twenty small-RBC blood samples with a mean corpuscular volume $(\mathrm{MCV})<70 \mathrm{fl}$ and 10 PLT aggregation samples were selected for PLT-I and PLT-O on the BC6000Plus. The PLT microscopy of the samples with small $\mathrm{RBC}$ interference was performed directly with the same tube of venous blood, while the microscopic PLT counting of the PLT aggregation samples required recollection of the peripheral blood samples for in vitro dilution and microscopic counting. The correlation between the Bc6000Plus and manual microscopy for samples with small $\mathrm{RBC}$ interference and PLT aggregation interference is shown in Table 3. The correlation of PLT-O was better than that of PLT-I. For PLT aggregation samples, the PLT-O correlated well with the recollection and dilution method.

\section{Discussion}

Accurate PLT counting is important for the diagnosis, treatment, and prognosis of blood diseases (7). Clinically, the risk of bleeding can be divided into different levels according to PLT count: a PLT count below $100 \times 10^{9} / \mathrm{L}$ indicates a risk of bleeding, a PLT count of $20-50 \times 10^{9} / \mathrm{L}$ indicates a possible risk of mild bleeding or surgical bleeding, a PLT count below $20 \times 10^{9} / \mathrm{L}$ indicates a high 
Table 2 Correlations among PLT counting results in different PLT ranges (r)

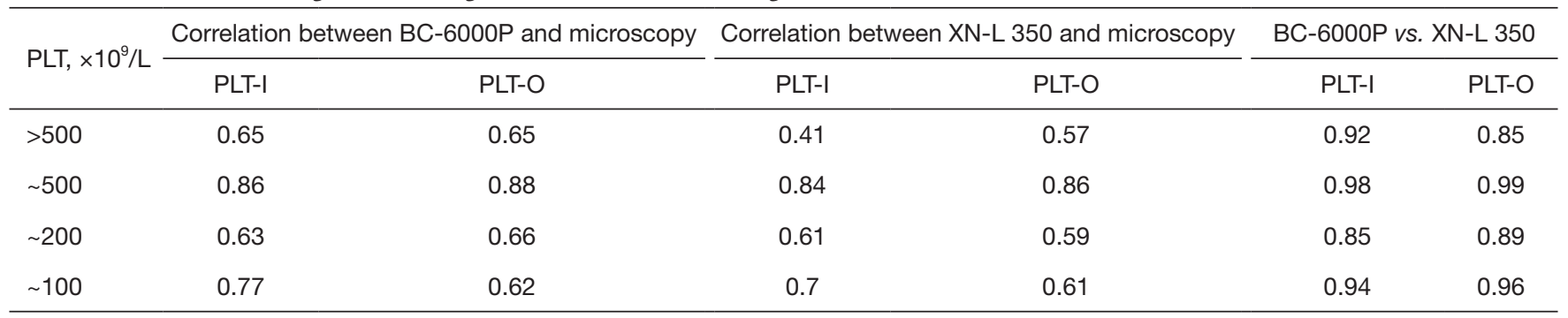

PLT, platelet; PLT-I, platelet impedance count; PLT-O, optical platelet counting.

Table 3 Correlations of PLT-I and PLT-O with manual microscopy when counting PLT in samples with small-RBC/platelet aggregate interference

\begin{tabular}{lcc}
\hline Type of interference & PLT-I & PLT-O \\
\hline MCV $<70 \mathrm{fl}$ (small red blood cell) & 0.86 & 0.87 \\
Platelet aggregation & 0.6 & 0.88 \\
\hline
\end{tabular}

PLT-I, platelet impedance count; PLT-O, optical platelet counting; PLT, platelet; RBC, red blood cell; MCV, mean corpuscular volume.

bleeding risk (8). In patients with thrombocytopenia, an accurate PLT count is critical for clinical decisionmaking. PLT transfusions are required when patients present with splenomegaly, coagulation factor deficiency, rapidly decreasing PLT count, or severe bleeding, among other indicators (9). As the blood cell analysis technology continues to advance, there is a growing clinical need for faster, more accurate, and more precise PLT counting methods. The impedance method (PLT-I) is the most commonly used method for PLT counting but has certain limitations (10). If other particles are present that are about the same size as PLTs in the blood, the instrument cannot correctly distinguish PLTs from such particles because it only uses high or low pulses to identify large or small particles (11). Clinically, interferences such as PLT aggregation and giant PLTs can cause a false decrease in PLT counts on PLT-I; meanwhile, interferences such as small RBCs, RBC fragments, and WBC fragments can cause a false increase in PLT counts on PLT-I (11). To compensate for the shortcomings of PLT-I, various additional methods have been developed by different manufacturers to doublecheck the PLT count. The Siemens ADVIA series, Abbott CELL-DYN series, and others have adopted the optical method (optical PLT, PLT-O) for PLT counting, which improves the detection of large PLTs but still has weak performance in overcoming the interference from small RBCs or cellular debris (12). Based on the optical method, the BC-6800 series (Mindray, Shenzhen, China) and the $\mathrm{XE}$ and $\mathrm{XN}$ series (Sysmex, Japan) have been developed. These new instruments, augmented by a specific ability to identify nucleic acids by nucleic acid fluorescent dyes, have greatly improved the anti-interference ability against small RBCs and cellular debris $(5,6)$. In recent years, novel nucleic acid fluorescent dyes that are more specific for PLT staining have been developed and have begun to be used clinically, receiving increasing attention from laboratory technicians. The representative methodologies include the enhanced PLT-O (PLT-O 8x) on the BC-6800Plus series (Mindray, Shenzhen, China) and the PLT-F on the XN series (Sysmex, Japan) $(13,14)$. At essence, these techniques remain optical methods; however, with the use of new nucleic acid fluorescent dyes and improved algorithms, they can dramatically increase the counting volume by $5-8$ times. This is particularly important for low PLT samples as it can greatly improve the precision of PLT detection at low levels. The PLT-O and PLT-O $8 \times$ on the BC-6000Plus serial hematology analyzers have the same principle and algorithm as the BC-6800Plus serial hematology analyzers. The difference is that the BC-6000Plus uses less blood, whereas the BC-6800Plus is faster. In our current study, we evaluated the performance of the PLT-O on the BC6000Plus serial hematology analyzers in routine PLT counting and in detecting abnormal PLT samples.

The blank counting, carryover rate, trueness, and accuracy of the BC-6000Plus hematology analyzer met the Analytical Quality Specifications for Routine Tests in Clinical Hematology (WST 406-2012), indicating that the analyzer can meet the performance requirements for basic clinical applications. Although the performance verification of low PLT count $\left(<100 \times 10^{9} / \mathrm{L}\right)$ is not considered as a routine quality requirement, when a test report on a low-PLT 
sample is required by a clinical department, the clinical laboratory should ensure the accuracy and repeatability of such a report. We divided the low-PLT samples into three ranges, including $\leq 20,20-60$, and $60-100\left(\times 10^{9} / \mathrm{L}\right)$ and found that the PLT-O $8 \times$ on the BC-6000Plus showed good repeatability in all three ranges, with a significantly smaller robust $\mathrm{CV}$ (\%) than that of the optical method on the XN-L 350. To further verify the correlation between the optical PLTs of BC-6000Plus hematology analyzer and the PLTs detected by the existing analytical system, the XN-L 350, PLT samples with four different ranges, 0-100, 100-200, 200-500, and $>500\left(\times 10^{9} / \mathrm{L}\right)$, were selected. It was found that the BC-6000Plus correlated better with the $\mathrm{XN}-\mathrm{L} 350$ than with manual microscopy, probably due to the poor repeatability of the microscopic method. In fact, manual microscopy is mainly used for the rechecking of abnormal PLT samples rather than as a reference method for PLT counting.

In addition to the low PLT count, PLT counting can also be made challenging by interference from Non-platelet particulates and PLT aggregation. In our current study, 20 small RBC samples with $\mathrm{MCV}<70 \mathrm{fl}$ were selected to identify PLT-O's anti-interference against small RBC. With the manual microscopy as a reference, it was found that, on the BC-6000Plus, PLT-I and PLT-O in particular correlated well with the manual microscopy. And PLT-O showed better consistency, which can be explained that fluorescent nucleic acid dye could stain PLT rather than RBC. In addition, 10 samples with PLT aggregation were selected in our study to identify PLT-O's anti-interference against platelet aggregation. Using manual microscopy after recollection and dilution, we found that the PLT-I on BC-6000Plus correlated poorly with manual microscopy counting after dilution. The possible explanations for this may be that (I) PLT aggregation causes a false low PLT-I counting and that (II) PLT aggregation leads to increased heterogeneity of PLT particles, which also decreases the PLT-I repeatability. However, PLT-O showed a better correlation with the post-dilution manual microscopy, which may be related to the "self-deaggregation" function of the PLT-O on the BC-6000Plus. It has been demonstrated that PLT-O on the BC-6800 and BC-6800Plus has a "deaggregation" effect on EDTA-induced aggregation of PLTs $(15,16)$ : the aggregated PLTs are disaggregated by the action of certain factors (e.g., deaggregating agent, temperature, and mixing) in the optical PLT channel; therefore, the counting result is very close to the PLT count in blood samples without PLT aggregation that are recollected after changing the anticoagulant. No previous study has described a similar "self-deaggregation" effect of PLT-O on the BC-6000Plus, although BC-6000Plus uses the same optical PLT detection system as the BC-6800Plus. It theoretically can also induce a "self-deaggregation" effect on EDTA-dependent samples with PLT aggregation. Our current study demonstrated that the PLT-O on the BC-6000Plus correlated well with post-dilution manual microscopy when counting PLT in 10 PLT aggregation samples, indicating that the PLT-O on the BC-6000Plus has the same "self-deaggregation" function for PLT aggregation samples.

In summary, we evaluated the basic performance of the optical PLT method of the BC-6000 Plus (Mindray, Shenzhen, China), a five-part hematology analyzer, and assessed its performance in detecting samples with interferences from small RBCs and PLT aggregation. We can conclude that the PLT-O on the BC-6000 Plus has (I) good essential functions in terms of blank count, carryover rate, trueness, and accuracy; (II) good anti-interference ability for small RBCs and PLT aggregation; and (III) good correlation with an existing detection system, the XN-L 350. The BC-6000 Plus thus meets the clinical requirements for PLT counting.

\section{Acknowledgments}

Funding: None.

\section{Footnote}

Reporting Checklist: The authors have completed the MDAR checklist. Available at http://dx.doi.org/10.21037/atm-21691

Data Sharing Statement: Available at http://dx.doi. org/10.21037/atm-21-691

Conflicts of Interest: All authors have completed the ICMJE uniform disclosure form (available at http://dx.doi. org/10.21037/atm-21-691). The authors have no conflicts of interest to declare.

Ethical Statement: The authors are accountable for all aspects of the work in ensuring that questions related to the accuracy or integrity of any part of the work are appropriately investigated and resolved. This study was approved by the ethics committee of The Maternal and Child Health Hospital of Guangxi Zhuang Autonomous 
Region. (No. [2021]2-1). All procedures performed in this study involving human participants were in accordance with the Declaration of Helsinki (as revised in 2013). Informed consent was obtained from the patients.

Open Access Statement: This is an Open Access article distributed in accordance with the Creative Commons Attribution-NonCommercial-NoDerivs 4.0 International License (CC BY-NC-ND 4.0), which permits the noncommercial replication and distribution of the article with the strict proviso that no changes or edits are made and the original work is properly cited (including links to both the formal publication through the relevant DOI and the license). See: https://creativecommons.org/licenses/by-nc-nd/4.0/.

\section{References}

1. International Council for Standardization in Haematology Expert Panel on Cytometry; International Society of Laboratory Hematology Task Force on Platelet Counting. Platelet counting by the RBC/platelet ratio method. A reference method. Am J Clin Pathol 2001;115:460-4.

2. Harrison P, Ault KA, Chapman S, et al. An interlaboratory study of a candidate reference method for platelet counting. Am J Clin Pathol 2001;115:448-59.

3. Harrison P, Horton A, Grant D, et al. Immunoplatelet counting: a proposed new reference procedure. $\mathrm{Br} \mathrm{J}$ Haematol 2000;108:228-35.

4. Chen XM, Liang JC. The clinical value of microscopic counting method in reviewing PLT counting of hematology analyzer. China Medicine and Pharmacy 2018;008:155-7.

5. Sun $Y, H u Z$, Huang $Z$, et al. Compare the accuracy and precision of Coulter LH780, Mindray BC-6000 Plus, and Sysmex XN-9000 with the international reference flow cytometric method in platelet counting. PLoS One 2019;14:e0217298.

6. Sun Y, Xiang DJ, Ye B, et al. Clinical application of new hematology analysis methodology SF-Cube 2.0. Medical

Cite this article as: Hu X, Tang Y, Li M, Fu C, Deng Z, Tang W, Zhou H, Chen Y, Li Y. Performance evaluation of optical platelet counting by BC-6000Plus automated hematology analyzer. Ann Transl Med 2021;9(6):508. doi: 10.21037/atm-21-691
Laboratory Science and Clinics 2018;15:3576-8.

7. Lippi G, Favaloro EJ, Buoro S. Platelet Transfusion Thresholds: How Low Can We Go in Respect to Platelet Counting? Semin Thromb Hemost 2020;46:238-44.

8. He L. Clinical significance of decreased platelet count. Guide of China Medicine 2009;7:123-4.

9. Ancliff PJ, Machin SJ. Trigger factors for prophylactic platelet transfusion. Blood Rev 1998;12:234-8.

10. C Tantanate, L Khowawisetsut, K Pattanapanyasat. Performance Evaluation of Automated Impedance and Optical Fluorescence Platelet Counts Compared With International Reference Method in Patients With Thalassemia. Arch Pathol Lab Med 2017;141:830-6.

11. M Zandecki, F Genevieve, J Gerard, et al. Spurious counts and spurious results on haematology analysers: a review. Part I: platelets. Int J Lab Hematol 2007;29:4-20.

12. Gresele P, Kleiman NS, Lopez JA, et al. Platelets in Thrombotic and Non-Thrombotic Disorders. Platelets in thrombotic and non-thrombotic disorders 2002; Cambridge University Press. 2002.

13. Schoorl M, Schoorl M, Oomes J, et al. New fluorescent method (PLT-F) on Sysmex XN2000 hematology analyzer achieved higher accuracy in low platelet counting. Am J Clin Pathol 2013;140:495-9.

14. Gioia M, Da Rin G, Manenti B, et al. Multicenter evaluation of analytical performances of platelet counts and platelet parameters: Carryover, precision, and stability. Int J Lab Hematol 2020;42:552-64.

15. Deng J, Chen Y, Zhang S, et al. Mindray SF-Cube technology: An effective way for correcting platelet count in individuals with EDTA dependent pseudo thrombocytopenia. Clin Chim Acta 2020;502:99-101

16. Bao Y, Wang J, Wang A, et al. Correction of spurious low platelet counts by optical fluorescence platelet counting of BC-6800 hematology analyzer in EDTA-dependent pseudo thrombocytopenia patients. Transl Cancer Res 2020;9:166-72.

(English Language Editor: J. Gray) 